\title{
An Ontological Evaluation of the OML Metamodel
}

\author{
Andreas L Opdahl ${ }^{1}$, Brian Henderson-Sellers ${ }^{2}$ and Franck Barbier ${ }^{3}$ \\ ${ }^{1}$ Department of Information Science, University of Bergen, Norway; andreas@ifi.uib.no . \\ Presently at the School of Computing Sciences, University of Technology, Sydney, Australia. \\ ${ }^{2}$ School of Computing Sciences, University of Technology, Sydney, Australia; \\ brian@socs.uts.edu.au. \\ ${ }^{3}$ IRIN, Université de Nantes, France; Franck.Barbier@irin.univ-nantes.fr.
}

Key words: Object-oriented modelling, Ontological analysis and evaluation, OPEN, OML, the BWW-model, FRISCO.

\begin{abstract}
The paper presents results of analysing and evaluating the OPEN Modelling Language (OML) metamodel in terms of the Bunge-Wand-Weber (BWW) model of information systems and the Framework of Information Systems Concepts (FRISCO). The analysis and evaluation has suggested several ways to improve the modelling constructs supported by OML and to improve how OML is defined and organised. It has also made it clearer how and why ontological models like the BWW-model and FRISCO can and should be used to improve and define object-oriented (OO) modelling languages. In particular, it has led to a template for defining $O O$ modelling constructs in terms of what they represent in the problem domain during IS development. Finally, the analysis and evaluation has suggested ways to improve FRISCO as an analysis and evaluation tool for $\mathrm{OO}$ modelling languages.
\end{abstract}

\section{INTRODUCTION}

Object-oriented (OO) modelling languages have become widespread in recent years. However, some critics claim that object orientation is less well suited for modelling "real-world" problem domains during information systems (IS) development because object-oriented abstractions are tailored to represent software artefacts rather than things in or aspects of problem domains. For example, critics question whether OO-concepts such as message passing and encapsulation are really features of the real-world 
things that are represented and whether things really belong to fixed classes throughout their life-times (e.g., Jackson, 1995; Parsons and Wand, 1997b.)

To shed light on these problems and related ones, the paper presents results of using ontology to analyse and evaluate how well the metamodel of the OPEN ${ }^{1}$ Modelling Language (OML; Firesmith et al., 1998) supports representation of "real-world" problem domains. The analysis and evaluation is anchored in the Bunge-Wand-Weber (BWW) model of information systems (e.g., Wand and Weber, 1995; Weber, 1997) and in the Framework of Information Systems Concepts (FRISCO; Falkenberg et al., 1998.) It is the result of systematically comparing more than 160 OML-constructs with 44 ontological constructs in the BWW-model and 98 constructs in FRISCO.

Since space does not permit a detailed discussion of each OMLconstruct, the paper instead discusses important outcomes in general. (1) The analysis and evaluation has suggested several ways to improve the modelling constructs supported by OML and to improve how OML is defined and organised. (2) It has made it clearer how and why ontological models like the BWW-model and FRISCO can and should be used to improve and define object-oriented $(\mathrm{OO})$ modelling languages. (3) As a consequence, it has led to a template for defining $\mathrm{OO}$ modelling constructs in terms of what they represent in the problem domain of IS development. (4) Finally, the analysis and evaluation has suggested ways to improve FRISCO as an analysis and evaluation tool for OO modelling languages. Since detailed suggestions for improving OML will be presented elsewhere, the paper will focus on the latter three outcomes.

In what follows, Section 2 first introduces OML and the BWW-model along with the method of ontological analysis and evaluation. Also, this section explains why we have chosen OML as our object of study and why we have chosen ontological analysis and evaluation based on the BWWmodel and FRISCO as our method of research. Section 3 then presents an outline of the analysis, before Section 4 gives an overview of our results. Finally, Section 5 concludes the paper and outlines paths for further work.

\section{THEORETICAL BACKGROUND}

This section will briefly present the OPEN Modelling Language (OML) and the Bunge-Wand-Weber (BWW) model of information systems. It will also explain our choices of study-object and research method.

The OML Metamodel. The OPEN ${ }^{1}$ Modelling Language (OML; Firesmith et al., 1998; Firesmith and Henderson-Sellers, 1998; HendersonSellers and Firesmith, 1998) is an object-oriented modelling language which provides modelling constructs, diagram types, graphical notations and a 
metamodel for representing problem domains as well as logical and physical IS designs during all phases of IS development. This paper focusses on OML's metamodel.

Although it is an independent modelling language with a user-base of its own, OML is perhaps more important as a variant of the Object Management Group (OMG)'s standard Unified Modelling Language (UML; OMG, 1999.) In OML, more sophisticated and more formal concepts can be evaluated in theory and in practice (Henderson-Sellers et al., 1999.) It has been argued that OML is more "properly OO" than UML and provides a richer set of modelling constructs (Graham, 1998.) Also, OML has fewer modelling constructs and appears better organised than UML. We consider OML an appropriate object of study for the following reasons: (1) Improvements to OML also contribute to the further development of UML. (2) Since OML is a "proper" and rich $\mathrm{OO}$ modelling language, our results can be readily generalised to OO modelling in general. (3) Since OML's metamodel is small and well organised, it is a useful intermediate step before analysing and evaluating the larger and more complex UML. Based on the results and experiences from the present study, we have initiated such a study of UML.

Since space does not allow us to present the OML metamodel in detail, we will explain each OML-construct we have used when it is encountered in the text.

Ontological Analysis and Evaluation. Ontological analysis and evaluation has been proposed by Wand and Weber (e.g., Wand and Weber, 1993) as a method for assessing and improving IS modelling languages. The method applies philosophical ontology to analyse the meaning of modelling constructs and to evaluate whether the set of constructs provided by a language is appropriate or not. Philosophical ontology can be described as "the science concerned with the whole of reality" (Bunge, 1977, p.5) and is therefore an appropriate foundation for analysing and evaluating modelling languages which are meant to represent "real-world" problem domains. (Although it is considerably more specialised due to its closer adaptation to the IS field, this paper will also treat FRISCO as an ontology in this sense.)

The method proposed by Wand and Weber prescribes two mappings between an ontological model (such as the BWW-model and FRISCO) and a modelling language to be evaluated (such as OML's metamodel.) On the one hand, an interpretation mapping from the set of modelling constructs to the set of ontological constructs is carried out to identify (1a) excessive modelling constructs with no ontological counterpart, as well as (1b) overloaded modelling constructs with more than one counterpart in the ontology. The interpretation mapping also provides (1c) a precise definition of each modelling construct in terms of the ontological model. On the other hand, a representation mapping from the set of ontological constructs to the 
set of modelling constructs is carried out to identify (2a) redundant modelling constructs, several of which have the same counterpart in the ontology, and (2b) deficits in the modelling language because there are ontological constructs with no counterpart in the language.

Our analysis and evaluation focusses only on OML's support for modelling "real-world" problem domains during IS development. We must therefore distinguish between two groups of excessive OML-constructs. One group is "truly excessive" because the constructs are clearly meant to represent something in the problem domain, yet have no counterparts in the ontology. Another group of excessive constructs is instead "not oriented towards problem domains" because the constructs are not meant to represent anything in the problem domain but instead represent, e.g., a part or aspect of the proposed software system. Only a "truly excessive" modelling construct is a problem.

We consider ontological analysis and evaluation an appropriate research method for the following reasons: (1) The analysis and evaluation has suggested several concrete improvements to OML and FRISCO. (2) It has prepared for a later analysis and evaluation of the OMG-standard, UML, and has suggested several other paths for further work.

The BWW-model. In addition to FRISCO, our ontological analysis and evaluation is anchored in the Bunge-Wand-Weber (BWW) model of information systems. The BWW-model has been proposed by Wand and Weber (e.g., Wand and Weber, 1995; Weber, 1997) as an adaptation to the IS field of Mario Bunge's comprehensive ontology (Bunge, 1977, 1979.)

We consider the BWW-model and FRISCO appropriate for our ontological analysis and evaluation of OML since they are two among several ontologies that can and should be used to improve and better define existing OO modelling languages. Following (Wand and Weber, 1993), we also argue that the BWW-model is (1) better developed and formalised than competing ontologies, (2) based on concepts that are fundamental to the computer science and IS fields and (3) productive, in the sense that it has produced useful results already. Also, the BWW-model has already been used to analyse and evaluate several other modelling languages and frameworks (e.g., Wand and Weber, 1989; Weber and Zhang, 1996; Green, 1996; Weber, 1997; Green and Rosemann, 1999.) In addition, our analysis and evaluation is based on earlier work by Wand (Wand, 1989) which has proposed the BWW-model as a formal model of objects. This work has resulted in an object-oriented information systems model (Takagaki and Wand, 1991) and in analyses and discussions of object-oriented concepts (Parsons and Wand, 1997a, 1997b.) However, to our knowledge, the work described here is the first analysis and evaluation of an existing objectoriented modelling language using the BWW-model. 
Since space does not allow us to present the BWW-model in detail, we will explain each BWW-construct we have used when it is encountered in the text.

\section{OUTLINE OF THE ANALYSIS}

Tables 1 to 3 show interpretation mappings of the 54 constructs in OML that are most important for representing problem domains. Since the full analysis is too large to be presented in detail, this section will instead present an overview and explain the OML-, BWW- and FRISCO-constructs we will use later. The next section will present and discuss the main results.

Objects and classes in OML. In relation to the BWW-model, OMLobjects represent BWW-things and OML-classes represent BWW-classes of things. In relation to FRISCO, OML-objects represent FRISCO-predicated things and OML-classes represent FRISCO-populations thereof. Hence both ontologies contain constructs which underpin OML's objects and object classes. However, whereas BWW-things are "concrete" things in the problem domain, FRISCO-predicated things are "conceptions" in the minds of human actors. This is because the BWW-model is based on "metaphysical realism" whereas FRISCO is a "constructivist" ontology. As a consequence, the two ontologies lead to different interpretations of OML-objects and this difference in turn leads to similarly different interpretations of many other OML-constructs.

Characteristics and responsibilities in OML. OML's characteristics represent what an object "knows", what it "does" and which "rules" it obeys. In the BWW-model, the three kinds of OML-characteristics represent "regular" BWW-properties, to BWW-transition laws and to BWW-state laws, respectively. (BWW-laws are a kind of BWW-properties.) In FRISCO, the corresponding three constructs are FRISCO-predicators "in general", FRISCO-rules about permissible transitions and FRISCO-rules about permissible states.

An important distinction made in the BWW-model (Weber, 1997) is between BWW-human laws (e.g., traffic regulations, business policies) and BWW-natural laws (e.g., laws of physics.) Whereas the natural laws always hold, human laws are social constructs which can be broken. OML does not represent this distinction explicitly, although violations of human laws might be represented as OML-exceptions. The FRISCO report (Falkenberg et al., 1998) distinguishes between rules and organisational norms.

OML-responsibilities have no primitive counterparts in the BWW-model or in FRISCO and still need to be defined more precisely in relation to "realworld" problem domains. 
Relationships in OML. OML defines a comprehensive set of relationships between objects and classes (Firesmith and Henderson-Sellers, 1998.) For example, OML distinguishes between using and non-using linkages between OML-objects. A using linkage is one used to invoke OMLoperations in another object. OML-linkages represent FRISCO-relationships as well as BWW-mutual properties, i.e., BWW-properties that belong to more than one BWW-thing. OML-using linkages represent BWW-mutual properties which couple two BWW-events occurring in different things, so that one event causes the other because it changes the mutual property. There is no similar FRISCO-construct.

OML also defines several variants of whole-part relationships (Henderson-Sellers and Barbier, 1999.) In this respect, OML is more finegrained than both the BWW-model's whole-part relations and FRISCO's set membership. (BWW-whole-part relations are also a kind of BWWproperties.) On the other hand, OML and FRISCO lack explicit distinctions like the one in the BWW-model between resultant and emergent properties of composite BWW-things.

Interactions, messages and scenarios in OML. An OML-interaction represents a FRISCO-message transfer and a pair of BWW-events which are coupled by a mutual property. However, in FRISCO there is no distinction which underpins the one in OML between normal and exceptional interactions and transitions.

An OML-scenario represents a "contiguous set of interactions" and has no primitive counterparts in the BWW-model or in FRISCO.

States and transitions in OML. Both ontologies comprise constructs which underpin OML's states and transitions. But whereas OML- and BWW-states are defined in terms of property values, FRISCO-states are composite objects.

\section{DISCUSSION OF THE RESULTS}

Whereas detailed clarifications of and suggestions of improvements to OML will be presented elsewhere, this section will give an overview of the results and focus in particular on how ontological models like the BWWmodel and FRISCO can and should be used to improve and define $O O$ modelling languages.

Suggested improvements to OML. The analysis and evaluation has identified several cases of overloaded, redundant and genuinely excessive OML-constructs, as well as few deficits in OML. Prominent examples of redundant constructs are the group of OML-constructs which represent 
$B W W$-state laws and the group of constructs which represent $B W W$ transformation laws. In addition, there are several overlaps between constructs which deal with OML-interactions and -messages on the one hand and with OML-states and -transitions on the other. An example of a deficit is that OML lacks a clear distinction which represets the BWW-model's distinction between human laws and natural laws. In comparison to FRISCO, OML also lacks a counterpart to transition structures and to goals and goal pursuing actors.

Better definitions of OML-constructs. The analysis and evaluation has proposed definitions of the most important OML-constructs in terms of which things in and aspects of the problem domain each construct is meant to represent. At present, the OML-constructs are defined both in relation to the BWW-model and in relation to FRISCO. Below we suggest to align these two sets of definitions in the future.

A related suggestion is to redefine OML's diagram types to ensure that the diagrams used during the early phases of IS development only comprise OML-constructs that represent things in and aspects of problem domains.

The multiple roles of OML-constructs. The analysis and evaluation has identified four major roles and several sub-roles played by the modelling constructs in OML. Two of the major roles confirm the ones identified in (Wand, 1989; Parsons and Wand, 1997b.) The major roles are (a) representing things in and aspects of the problem domain, (b) representing things in and aspects of the development process, (c) representing things in and aspects of the final software system, and (d) contributing to and complying with the internal organisation of OML's metamodel. Whereas some OML-constructs play only one of these roles, others play two or more.

OML is one of the few $\mathrm{OO}$ modelling languages that has been developed with an explicit aim of representing "real-world" problem domains in addition to designs and implementations. Our study nevertheless indicates that many of its constructs are defined to compromise between these four roles and often at the expense of role (a). We therefore suggest that the four roles should be identified explicitly in the definition of each OML-construct. The internal organisation of OML's metamodel should be revised to reflect this.

Template for defining IS-modelling constructs. Our experience with interpreting and defining OML-constructs in terms of the two ontologies has lead to a template for defining modelling constructs in relation to "realworld" problem domains. We expect the template to be useful even for other modelling languages than $\mathrm{OML}, \mathrm{OO}$ or otherwise.

The template is presently based on the BWW-model. In particular, it is based on the fundamental view inherent in the BWW-model that the world is 
comprised of $B W W$-things and their $B W W$-properties. Although the BWWmodel introduces several other constructs, they all somehow emerge from temporal and other relations between these two fundamental ones. The template therefore requires each modelling construct to be defined by answering the following three questions:

1. Which class of things is the construct meant to represent?

2. Which property (or group of properties) is the construct meant to represent?

3. Which ontological category is the construct meant to represent?

The first question delimits each OML-construct in "thing space", i.e., with respect to which kind of things it is meant to represent. This question is answered by making explicit the characteristic properties of the BWW-class in question, i.e., the set of properties that is possessed by all the BWWthings in the class. For example, "OML-software external" is meant to represent BWW-things which are software artefacts and with which the proposed software system will interact. "OML-path", on the other hand, is meant to represent BWW-things which are systems of other BWW-things that are involved in some OML-scenario. In this way, different OMLconstructs are meant to represent different classes of things in the problem domain. A group of very general OML-constructs, which includes "OMLclass", "OML-object" and "OML-characteristic", are meant to represent any kind of BWW-things, regardless of their class.

The second question delimits each OML-construct further in "property space", i.e., with respect to which aspects of things it is meant to represent. When it is applicable to an OML-construct, this question is answered by making explicit the actual $B W W$-property or group of properties that the construct is meant to represent. For example, "OML-using linkage" is meant to represent BWW-mutual properties which couple two BWW-events that occur in different things. "OML-aggregation relationship", on the other hand, is meant to represent BWW-whole-part relations along with certain BWW-state laws that pertain to the whole and its parts. In this way, different OML-constructs are meant to represent different kinds of properties of things in the problem domain. Again, a group of general OML-constructs (which only includes "OML-characteristic") is meant to represent any kind of BWW-properties. For another group of general OML-constructs, the second question is not applicable. Constructs in this group, such as "OMLclass" and "OML-object", are not meant to represent BWW-properties at all.

However, a modelling language may even provide several modelling constructs meant to represent the same group of BWW-properties (for example, mutual properties) for the same class of BWW-things (for example, human actors). Although these modelling constructs are identical with respect to questions 1 and 2, they may still differ because they represent 
different ontological phenomena (for example, the values of mutual properties of humans, or their histories over time, or the events that occur in them etc.) The third question therefore delimits each modelling construct further in "ontology space", i.e., with respect to which ontological constructs it is meant to represent. In OML, "interaction" and "using linkage" are identical in all respects except in ontology space.

The template has already been used successfully to propose definitions of all the 54 most important OML-constructs. However, there may still be some overlap between the second and third questions and we plan to explore the template further by using it on other modelling languages in addition to OML.

The relation between the BWW-model and FRISCO. The ontological analysis and evaluation has also put us in a position where we can compare the two ontologies we have applied. On the one hand, FRISCO appears more coarse-grained than the BWW-model in relation to systems and to dynamic behaviour. For example, FRISCO does not distinguish between resultant and emergent predicators of FRISCO-composite things and it has no constructs which match the BWW-distinctions between external and internal events and between stable and unstable states ${ }^{2}$. However, FRISCO is also more finegrained than the BWW-model in a few areas. For example, it supports state transition structures (sequence, choice and concurrency) and it distinguishes between transitions as general behaviour patterns at the type level and transition occurrences at the instance level.

On the other hand, in other areas the BWW-model appears more general than FRISCO. For example, unlike FRISCO, the BWW-model lacks explicit constructs related to perceptions, conceptions, interpretations and representations. FRISCO also comprises several other specialised constructs which are relevant to the IS field, such as knowledge, models and modelling and which are not found in the BWW-model.

This explains our choice of the BWW-model, rather than FRISCO, as our starting point for defining the above template. The BWW-model is sometimes (but not always) more comprehensive than FRISCO in "ontology space", whereas FRISCO is more closely adapted to the IS field in "thing space." The BWW-model is therefore a better starting point because it can easily import FRISCO-constructs it lacks by simple specialisation in "thing space", whereas extending FRISCO with BWW-constructs it lacks in "ontology space" is more difficult.

Improvements to FRISCO. The ontological analysis and evaluation has so far suggested several improvements to and extensions of FRISCO. Concrete suggestions for extensions inspired by the BWW-model include FRISCO-constructs which distinguish between resultant and emergent predicators of composite things and constructs which distinguish between 
stable and unstable states as well as external and internal events. In addition, OML's metamodel and related work identify numerous useful distinctions between different kinds of whole-part and other relationships (HendersonSellers and Barbier, 1999; Firesmith and Henderson-Sellers, 1998.) Another challenging task is to clarify the semantics of responsibilities of human and other actors within FRISCO.

Our comparison has shown that the BWW-model and FRISCO each have their comparative advantages. Since the two differ in ontological coarseness and degree of adaptation to the IS field, an interesting path for further work is to reconcile the two, e.g., using the proposed template to define each FRISCO-construct in terms of the BWW-model.

This raises the question of how an ontology based on "metaphysical realism" such as the BWW-model can be reconciled with a "constructivist" framework such as FRISCO. We argue that this is possible because the BWW-model distinguishes between properties that are inherent in BWWthings and attributes that are ascribed to models of BWW-things. We only know things in the world through models we make of these things, and we ascribe attributes to model things in order to represent the properties we believe the real things have (Bunge, 1977, pp. 58-60; Weber, 1997.) Since an attribute is dependent on both the observer thing and the thing observed, it therefore turns out to be a $B W W$-mutual property of the two or a $B W W$ emergent property of the BWW-system of the two. We conjecture that all the FRISCO-constructs that are "conceptions" of "domains" can be analysed in terms of the BWW-model in this way. Similar ideas have been elaborated as part of the facet-modelling framework (Opdahl and Sindre, 1997) which may guide reconciliation of the BWW-model and FRISCO along the lines suggested here.

\section{CONCLUSION AND FURTHER WORK}

The paper has presented an ontological analysis and evaluation of the OPEN Modelling Language in terms of the BWW-model and FRISCO. The analysis and evaluation has made it clearer how and why ontological models like the BWW-model and FRISCO can and should be used to improve and define object-oriented $(\mathrm{OO})$ modelling languages. As a consequence, it has led to a template for defining OO modelling constructs in terms of what they represent in the problem domain during IS development. The analysis and evaluation has also suggested ways to improve FRISCO as an analysis and evaluation tool for $\mathrm{OO}$ modelling languages. Although they have not been presented in this paper, the analysis and evaluation has also suggested 
several ways to improve the modelling constructs supported by OML and to improve how OML is defined and organised.

There are several paths for further work in addition to the ones mentioned already. For one thing, the analysis has only considered "conventional" OOconstructs - such as objects, classes, operations, etc. - which are already part of OML. An interesting further development is to extend OML with a broader spectrum of constructs from the requirements engineering field, where constructs such as agents, goals and obstacles are currently topical. The two research areas already overlap in their shared focus on scenarios, interactions, actors and roles.

Also, whereas this study has focussed on the individual modelling constructs in OML, another interesting path is to evaluate the seventeen diagram types it supports. Such an analysis could form the basis of an empirical study of how OML-diagrams are used in practice, along the lines suggested in (Green, 1996.)

Finally, we will follow-up this study by analysing and evaluating the Unified Modeling Language (UML; OMG, 1999) in a similar way and eventually compare the two languages.

\section{ACKNOWLEDGMENTS}

This is Contribution number $99 / 8$ of the Centre for Object Technology Applications and Research (COTAR). Andreas L. Opdahl wishes to thank COTAR and the University of Technology, Sydney, as well as the Department of Commerce, University of Queensland where parts of this work was carried out.

\section{NOTES}

1. Object-oriented Process, Environment, and Notation (OPEN) is an integrated objectoriented information systems development methodology (Graham et al., 1997; HendersonSellers et al., 1998.)

2. BWW-external event: "An event that arises in a thing, subsystem or system by virtue of the action of some thing in the environment of the thing, subsystem or system." $B W W$-internal event: "An event that arises in a thing, subsystem or system by virtue of lawful transformations in the thing, subsystem or system." BWW-unstable state: "A state that will be changed into another state by virtue of the action of transformation in the system." $B W W$-stable state: "A state in which a thing, subsystem or system will remain unless forced to change by virtue of the action of a thing in the environment (an external event)" (Wand and Weber, 1995.) 


\section{REFERENCES}

Bunge, M. (1977). Treatise on Basic Philosophy: Vol. 3: Ontology I: The Furniture of the World. Reidel, Boston.

Bunge, M. (1979). Treatise on Basic Philosophy: Vol. 4: Ontology II: A World of Systems. Reidel, Boston.

Falkenberg, E. D., Hesse, W., Lindgreen, P., Nilsson, B. E., Oei, J. L. H., Rolland, C., Stamper, R. K., Van Assche, F. J. M., Verrijn-Stuart, A. A., and Voss, K. (1998). A Framework of Information System Concepts - The FRISCO Report (Web edition). IFIP.

Firesmith, D. and Henderson-Sellers, B. (1998). Upgrading OML to version 1.1: Part 1 referential relationships. Journal of Object-Oriented Programming (ROAD), 11(3):48-57.

Firesmith, D., Henderson-Sellers, B., and Graham, I. (1998). OPEN Modelling Language OML Reference Manual. SIGS Reference Library. Cambridge University Press.

Graham, I. (1998). Requirements Engineering and Rapid Development - An Object-Orientea Approach. Addison-Wesley.

Graham, I., Henderson-Sellers, B., and Younessi, H. (1997). The OPEN Process Specification. Addison-Wesley, U.K. 314 pp.

Green, P. and Rosemann, M. (1999). An ontological evaluation of integrated process modelling. Proceedings of CAiSE*99, The 11th Conference on Advanced information Systems Engineering, Heidelberg/Germany, 14-18 June 1999.

Green, P. F. (1996). An Ontological Analysis of Information Systems Analysis and Design (ISAD) Grammars in Upper CASE Tools. PhD thesis, Department of Commerce, University of Queensland.

Henderson-Sellers, B., Atkinson, C., and Firesmith, D. G. (1999). Viewing the OML as a variant of the UML. To be presented at $<<\mathrm{UML}^{\prime} 99>>$, Fort Collins/CO.

Henderson-Sellers, B. and Barbier, F. (1999). What is this thing called aggregation? In Mitchell, R., Wills, A. C., Bosch, J., and Meyer, B., editors, TOOLS29, pages 216-230. IEEE Computer Society Press.

Henderson-Sellers, B. and Firesmith, D. (1998). Upgrading OML to version 1.1: Part $2-$ additional concepts and notation. Journal of Object-Oriented Programming (ROAD), 11(5):61-67.

Henderson-Sellers, B., Simons, A. J. H., and Younessi, H. (1998). The OPEN Toolbox of Techniques. Addison-Wesley, U.K. 426 pp. + CD.

Jackson, M. (1995). Software Requirements \& Specifications - A lexicon of practice, principles and prejudices. ACM Press/Addison-Wesley, Wokingham/England.

OMG (1999). OMG Unified Modeling Language Specification.

Opdahl, A. L. and Sindre, G. (1997). Facet modelling: An approach to flexible and integrated conceptual modelling. Information Systems, 22(5):291-323.

Parsons, J. and Wand, Y. (1997a). Choosing classes in conceptual modeling. CACM, 40(6):63-69. 
Parsons, J. and Wand, Y. (1997b). Using objects for systems analysis. CACM, 40(12):104 110.

Sommerville, I. and Sawyer, P. (1997). Requirements Engineering - A good practice guide. Wiley, Chichester/England.

Takagaki, K. and Wand, Y. (1991). An object-oriented information systems model based on ontology. In Van Assche, F., Moulin, B., and Rolland, C., editors, Object Oriented Approach in Information Systems, pages 275-296, Amsterdam. Elsevier (North-Holland).

Wand, Y. (1989). A proposal for a formal model of objects. In Kim, W. and Lochovsky, F. H., editors, Object-Oriented Concepts, Databases, and Applications, chapter 21, pages 537-559. ACM Press/Addison-Wesley, New York/NY.

Wand, Y. and Weber, R. (1989). An ontological evaluation of systems analysis and design methods. In Falkenberg, E. and Lindgreen, P., editors, Proceedings of the IFIP WG8.1 Working Conference on Information Systems Concepts: An In-Depth Analysis, Namur/Belgium, pages 79-107, Amsterdam/The Netherlands. North-Holland.

Wand, Y. and Weber, R. (1993). On the ontological expressiveness of information systems analysis and design grammars. Journal of Information Systems, 3:217-237.

Wand, Y. and Weber, R. (1995). On the deep structure of information systems. Information Systems Journal, 5:203-223.

Weber, R. (1997). Ontological Foundations of Information Systems. Number 4 in Accounting Research Methodology Monograph series. Coopers \& Lybrand, 333 Collins Street, Melbourne Vic 3000, Australia.

Weber, R. and Zhang, Y. (1996). An analytical evaluation of NIAM's grammar for conceptual schema diagrams. Information Systems Journal, 6:147-170. 
Table 1 When OML-constructs are taken to represent concrete problem domains, they map onto BWW-constructs. When taken to represent conceptions of problem domains, they map onto the corresponding FRISCO-constructs. (Part 1 of 3.)

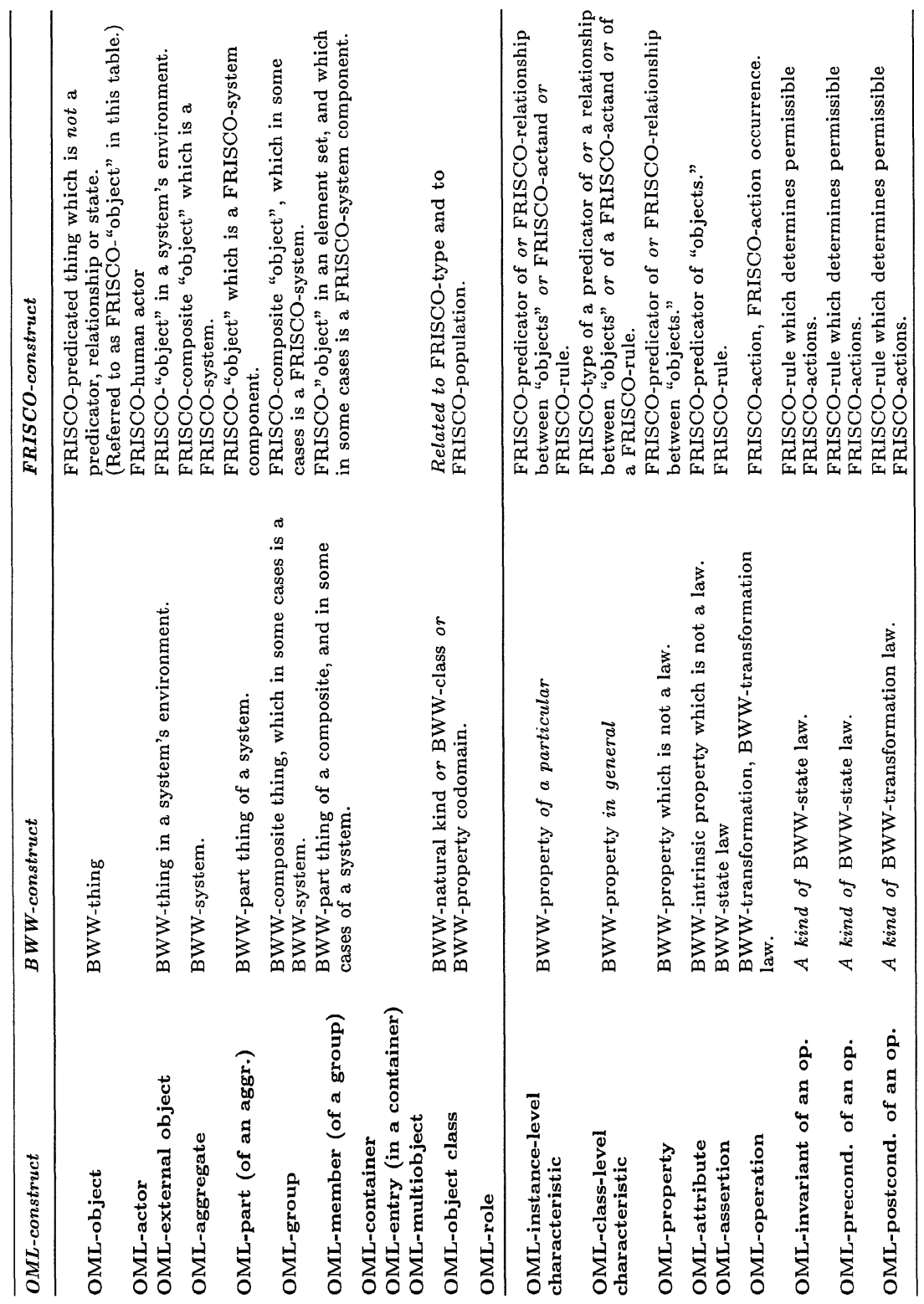


Table 2 When OML-constructs are taken to represent concrete problem domains, they map onto BWW-constructs. When taken to represent conceptions of problem domains, they map onto the corresponding FRISCO-constructs. (Part 2 of 3.)

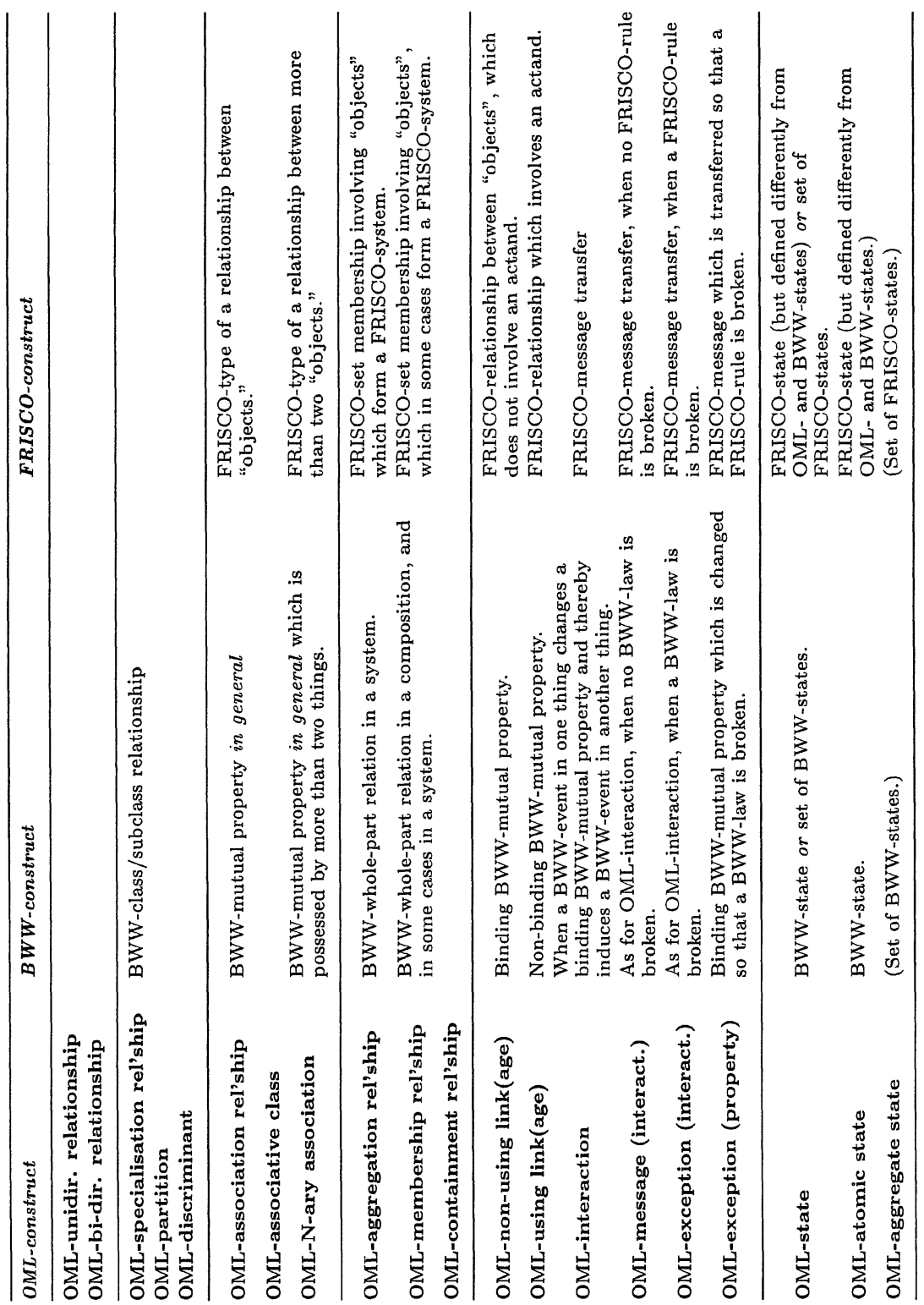


Table 3 When OML-constructs are taken to represent concrete problem domains, they map onto BWW-constructs. When taken to represent conceptions of problem domains, they map onto the corresponding FRISCO-constructs. (Part 3 of 3.)

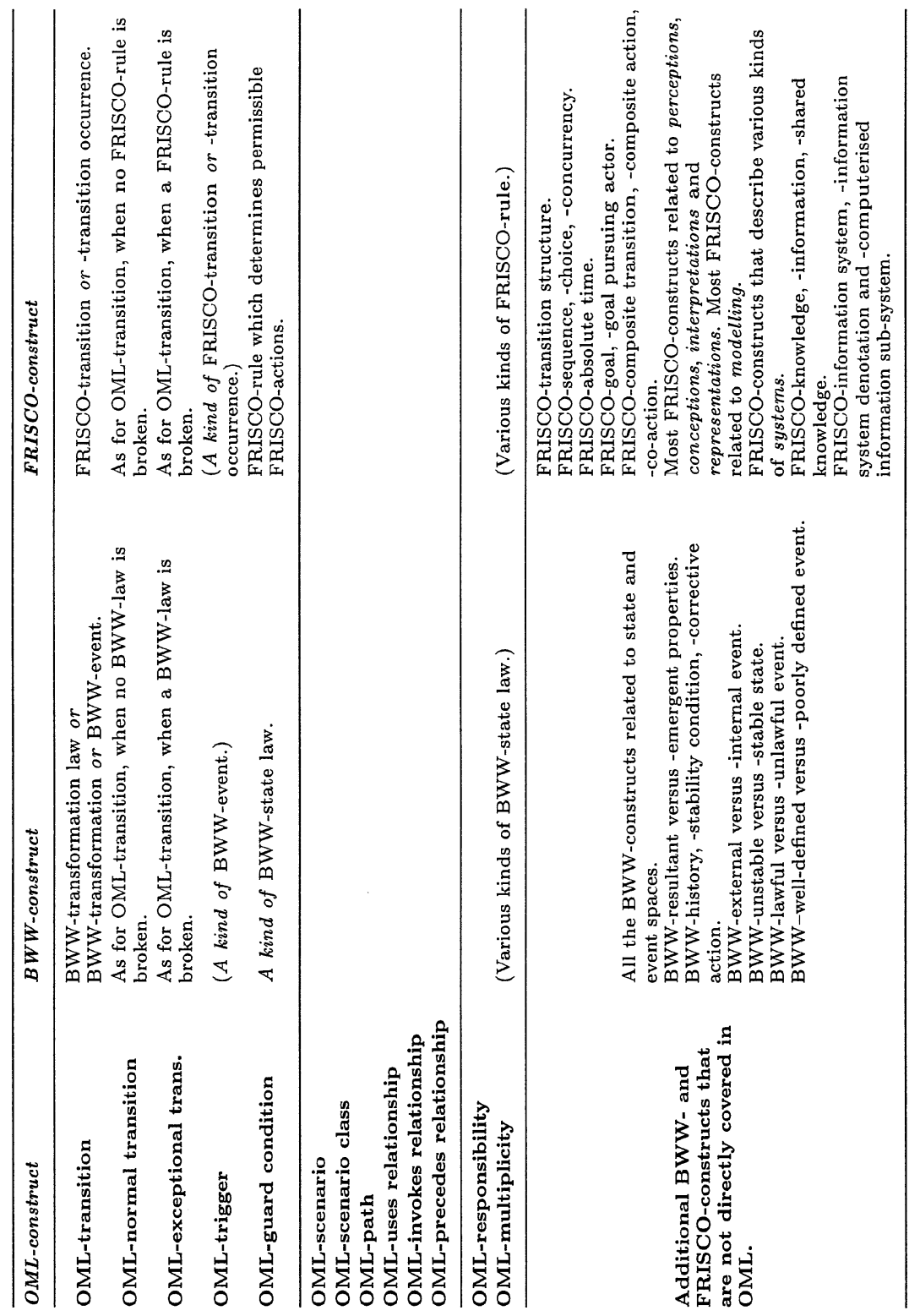

